\title{
О МНОГООБРАЗИЯХ ПРЕДСТАВЛЕНИЙ СВОБОДНЫХ АБЕЛЕВЫХ ГРУПП
}

\author{
А.А. Шаромет \\ Белгосуниверситет, механико-математический факультет \\ Независимости 4, 220050 Минск, Беларусь sharomet@mail.ru
}

Для многообразия $\mathcal{M} \subseteq M_{n}=M_{n}(P)$ определим многообразие перестановочных матриц

$$
C(m, \mathcal{M})=\left\{\left(a_{1}, \ldots, a_{m}\right) \in \mathcal{M} \mid a_{i} a_{j}=a_{j} a_{i}, i, j=\overline{1, m}\right\}
$$

которое можно интерпретировать как многообразие представлений свободной абелевой группы ранга $m[1,2]$. Обычно $\mathcal{M}$ - это $\mathrm{M}_{n}$ или линейная алгебраическая группа.

В качестве основной задачи рассматривается доказательство неприводимости многообразия $C(m, \mathcal{M})$ или описание его неприводимых компонент. Большое количество работ посвящено изучению многообразий $C\left(m, \mathrm{M}_{n}\right)$. Чтобы воспользоваться этими результатами для многообразий $C\left(m, \mathrm{GL}_{n}\right)$ и $C\left(m, \mathrm{SL}_{n}\right)$, рассмотрим взаимосязи между неприводимостью коммутаторных многообразий $C(m, n), C\left(m, \mathrm{GL}_{n}\right)$ и $C\left(m, \mathrm{SL}_{n}\right)$

Нетрудно убедиться, что $C\left(m, \mathrm{GL}_{n}\right)$ является открытым плотным множеством в $C\left(m, \mathrm{M}_{n}\right)$ и, значит они неприводимы одновременно.

Что касается многообразий $C\left(m, \mathrm{GL}_{n}\right)$ и $C\left(m, \mathrm{SL}_{n}\right)$, то их одновременная неприводимость следует из следующего более общего результата.

Теорема 1. Пусть $G=T H$ - почти прямое произведение одномерного тора $T$ и (связной) редуктивной группы $H$. Тогда многообразие $C(m, H)$ неприводимо тогда и толъко тогда, когда неприводимо многообразие $C(m, G)$.

Отметим последние результаты о неприводимости многообразий $C\left(m, \mathrm{M}_{n}\right)$, не претендуя на полноту обзора. Первый результат, состоящий в доказательстве неприводимости многообразия $C\left(2, M_{n}\right)$, содержится в известной работе [3]. Хорошо известно, что при $m, n \geq 4$ многообразие $C(m, n)$ приводимо, а при $n=3$ неприводимо при любом $m$. Значительное количество работ касается неприводимости $C(3, n)$; так, в работе [4] доказано, что $C(3, n)$ приводимо для $n \geq 32$. Авторы [5] заметили, что из рассуждений Гуральника в [4] легко следует, что $C(3, n)$ приводимо для $n \geq 29$. Наконец, в работе [6] доказано, что $C(3, n)$ неприводимо при $n \leq 10$ в предположении, что характеристика поля нулевая. Вопрос о неприводимости $C(3, n)$ при $10<n<29$ пока остается открытым.

Эти результаты, как показано выше, влекут за собой соответствующие следствия для односвязной группы $\mathrm{SL}_{n}$, которые мы соберем в следующее предложение. Поскольку в большинстве указанных работ характеристика основного поля предполагается нулевой, то и мы сохраним это ограничение.

Предложение. Многообразие $C\left(m, \mathrm{SL}_{n}\right)$ над полем нулевой характеристики неприводимо в случалх $1-3$ и приводимо в случалх 4,5 .

1. $m=2$, а $n$ - любое;

2. $n=3$, а $m$ - любое;

3. $m=3, n \leq 10$;

4. $m \geq 4, n \geq 4$;

5. $m=3, n \geq 29$.

Все сказанное выше можно считать развернутой мотивировкой задачи описания зависимости неприводимости многообразия $C(m, G)$ от параметра $m$ и ранга группы $G$ для односвязной группы. 
В работе [7] доказано, что для неодносвязной группы $G$ многообразие $C(m, G)$ не является неприводимым для $m \geq 2$, что вместе с теоремой Ричардсона [8] показывает, что над полем комплексных чисел односвязность группы $G$ является необходимым и достаточным условием неприводимости многообразия $C(2, G)$. Для неодносвязной группы $G$ остается найти неприводимые компоненты для $C(m, G)$. Если ограничиться случаем $m=2$, то естественными кандидатами на роль неприводимых компонент в $C(2, G)$ являются $p(W(\tilde{G}, \gamma))$, где $\pi: \tilde{G} \rightarrow G$ - универсальное накрытие, $p: \tilde{G} \times \tilde{G} \rightarrow G \times G-$ его квадрат, а $\gamma$ - элемент фундаментальной группы.

Таким образом, для неприводимости множеств $\theta^{-1}(\gamma)$ достаточно убедиться в неприводимости подмножества $p(W(\tilde{G}, \gamma))$, в частности, достаточно доказать неприводимость $W(\tilde{G}, \gamma)$. Единственный известный нам результат, касающийся неприводимости многообразия $W(\tilde{G}, \gamma)$ для односвязной группы, содержится в [5], и состоит в том, что многообразие

$$
W\left(\mathrm{SL}_{n}, \varepsilon\right)=\left\{(a, b) \in \mathrm{SL}_{n}^{2} \mid[a, b]=\varepsilon E\right\}
$$

неприводимо, если $\varepsilon$ - примитивный корень из единицы степени $n$. Отметим, что это решает задачу описания неприводимых компонент в многообразиях $C(2, G)$ для групп типа $A_{n}$, если $n+1$ - простое число.

Опишем неприводимые компоненты многообразия $C(2, G)$ для групп типа $A_{n}$ в общем случае. Через $\mathfrak{C}_{d}$ будем обозначать группу всех корней из 1 степени $d$. Основным результатом является следующая теорема.

Теорема 2. Пусть $G$ алгебраическал группа типа $A_{n}$, а $\pi: \mathrm{SL}_{n+1} \rightarrow G$-универсальное

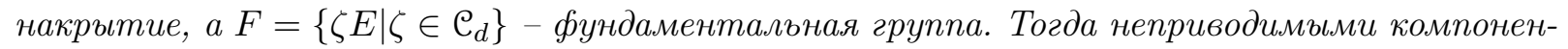
тами для $C(2, G)$ являются множества $p\left(W\left(\mathrm{SL}_{n}, \zeta\right)\right)$, где $\zeta \in \mathrm{C}_{d}$.

\section{Литература}

1. Lubotzky A., Magid A. Varieties of representations of finitely generated groups // Memoirs of the American Mathematical Society. 1985. V. 58. P. 1-116.

2. Платонов В.П., Рапинчук А. С. Алгебраические группъ и теория чисел. М.: Наука, 1991.

3. Motskin T., Taussky O. Pairs of matrices with property L. II // Trans. Amer. Math. Soc. 1955. V. 80. P. 387-401.

4. Guralnick R. A note on commuting pairs of matrices// Linear and Multilinear Algebra. 1992. V. 31. P. $71-75$.

5. Rapinchuk A. S., Benyash-Krivetz V. V., Chernousov V. I. Representation varietes of the fundamental grjups of cjmpact orientable surfases // Izrael J.of Math. 1996. V. 93, P. 29-71.

6. Šivic K. On varieties of commuting triples // Linear Algebra and its Applications. 2012. V. 437. P. 393-460.

7. Шаромет А.А. О многообразии пар перестановочных матрии, неодносвязной алгебрачческой группы // XI Белорусская математическая конференция: Тезисы докладов. Минск: Институт математики НАН Беларуси, 2012. Ч.5. С. 57-58.

8. Richardson R. W. Commuting varieties of semisimple Lie algebras and algebraic groups // Compositio Math. 1979. V. 38, No. 3. P. 311-327. 